\section{Production of tongue twisters by speakers with partial glossectomy} \\ Bressmann, T.; Foltz, A.; Zimmermann, J.; Irish, J.C.
}

\title{
Clinical Linguistics and Phonetics
}

\author{
DOI: \\ $10.3109 / 02699206.2014 .938833$
}

Published: 21/07/2014

Peer reviewed version

Cyswllt i'r cyhoeddiad / Link to publication

Dyfyniad o'r fersiwn a gyhoeddwyd / Citation for published version (APA):

Bressmann, T., Foltz, A., Zimmermann, J., \& Irish, J. C. (2014). Production of tongue twisters by speakers with partial glossectomy. Clinical Linguistics and Phonetics, 28(12), 951-964. https://doi.org/10.3109/02699206.2014.938833

\footnotetext{
Hawliau Cyffredinol / General rights

Copyright and moral rights for the publications made accessible in the public portal are retained by the authors and/or other copyright owners and it is a condition of accessing publications that users recognise and abide by the legal requirements associated with these rights.

- Users may download and print one copy of any publication from the public portal for the purpose of private study or research.

- You may not further distribute the material or use it for any profit-making activity or commercial gain

- You may freely distribute the URL identifying the publication in the public portal ?

\section{Take down policy}

This is an Accepted Manuscript of an article published by Taylor \& Francis in 'Clinical Linguistics \& Phonetics' on 21st of July 2014, available online: http://www.tandfonline.com/doi/full/10.3109/02699206.2014.938833

Take down policy

If you believe that this document breaches copyright please contact us providing details, and we will remove access to the work immediately and investigate your claim.
} 
Production of tongue twisters by speakers with partial glossectomy

\begin{abstract}
A partial glossectomy can affect speech production. The goal of the present study was to investigate the effect of the presence of a tumour as well as the glossectomy surgery on the patients' production of tongue twisters with the sounds $[\mathrm{t}]$ and $[\mathrm{k}]$. Fifteen tongue cancer patients and 10 healthy controls took part in the study. The outcome measures were the patients' speech acceptability, rate of errors, the time needed to produce the tongue twisters, pause duration between item repetitions, and the tongue shape during the production of the consonants $[\mathrm{t}]$ and [k] before and after surgery. The patients' speech acceptability deteriorated after the surgery. Compared to controls, the patients' productions of the tongue twisters were slower but not more errorful. Following the surgery, their speed of production did not change but the rate of errors was higher. Pause duration between items was longer in the patients than in the controls but did not increase from before to after surgery. Analysis of the patients' tongue shapes for the productions of $[\mathrm{t}]$ and $[\mathrm{k}]$ indicated a higher elevation following the surgery for the patients with flap reconstructions. The results demonstrated that the surgical resection of the tongue changed the error rate but not the speed of production for the patient. The differences in pause duration also indicate that the tumour and the surgical resection of the tongue may impact the phonological planning of the tongue twister.
\end{abstract}

Keywords

Glossectomy, tongue, tongue resection, speech production, speech errors, tongue twister, ultrasound 


\section{INTRODUCTION}

Glossectomy surgery is a common treatment approach for tongue cancer. Lingual cancer surgery will result in a defect of the tongue and change the orientation of its intrinsic muscles (Murano et al., 2010; Bressmann, Ackloo, Heng \& Irish, 2007). The effect on the patient's vowel space (Whitehill, Ciocca, Chan \& Samman, 2006) or consonants (Bressmann, Jacobs, Quintero \& Irish, 2009) depends on the defect size and location (Nicoletti et al., 2004). If the defect is closed locally (by suturing the wound margins), tongue motility may be affected. Alternatively, defects can be closed with free flaps such as radial forearm transplants. The advantage of a flap over a local closure is that the lost tongue tissue is replaced (Kimata et al., 2003), but some researchers argue that local closures lead to better speech outcomes (Konstantinovic \& Dimic 1998; Nicoletti et al. 2004) and that the flap tissue creates adynamic segments in the tongue (Bressmann et al., 2007).

After the glossectomy surgery, the structural changes to the tongue require the patient to adjust his or her articulatory gestures and to adapt to the altered anatomy. In partial glossectomy patients, the acceptability (i.e., the degree of normality) of speech will be more affected than speech intelligibility (Bressmann et al., 2009). While the presence of a lingual defect could lead one to expect that there should be less tongue movement and speed, Rastadmehr, Bressmann, Smyth and Irish (2008) found an increase of tongue height and speed during speech in patients with small to medium-sized partial glossectomies. In terms of speech motor planning, an imaging study in patients with glossectomy found increased activity in the parietal lobes and the cerebellum, which the authors interpreted as an indication of active compensatory adaptation to the structural defects (Mosier et al., 2005). Taken together, the studies by Rastadmehr et al. (2008) and Mosier et al. (2005) suggest that speakers with a partial glossectomy actively compensate for the structural defect to the tongue on the levels of speech planning and execution.

Previous studies on glossectomy speech have used commonplace speech tasks, such as word lists (Whitehill et al., 2006), sentences (Nicoletti et al., 2004) or text reading (Rastadmehr et al., 2008). However, to the best of the authors' knowledge, there has not been any research on the ability of partial glossectomy patients to carry out more challenging speech tasks. Such research might provide an insight into the speech planning and the compensatory strategies of patients 
with partial glossectomy. An example of a naturalistic speech task with high phonological and articulatory complexity are tongue twisters. The goal of the present study was to gather first empirical data on how speakers with a partial glossectomy cope with the production of tongue twisters.

Research has demonstrated that tongue twisters challenge both phonological planning and speech articulation. On the level of phonological planning, Oppenheim and Dell (2008, 2010) investigated errors in inner speech and in mouthed silent speech. Research participants silently reading a tongue twister were able to indicate where their phonological planning was affected. While their inner speech errors exhibited lexical bias, their mouthed errors were characterized by phonetic similarity effects. On the speech articulation level, research using electromagnetic articulography and ultrasound imaging of the tongue has shown that tongue twisters can cause confusion and discoordination of the articulators, such as parallel execution of conflicting articulatory gestures (Pouplier, 2007; Goldstein et al., 2007; McMillan \& Corley, 2010).

A tongue twister is a social word game that depends on the quick and fluent production of a phonologically and phonetically difficult sentence or text. The entertainment value of the tongue twister lies in the confusion and speech errors that it induces. In order to produce an acceptable rendition of a tongue twister, the speaker must balance speed and accuracy. The word game would no longer be amusing if the speaker produced the tongue twister too slowly or with complete disregard for the errors. Such speed-accuracy trade-offs are observed in various linguistic and non-linguistic tasks (e.g. Wickelgren, 1977; Goozee, Stephenson, Murdoch, Darnell, \& Lapointe, 2005). In a task that places demands on both speed and accuracy, speakers compensate for either speed or accuracy, but not both (Wickelgren, 1977). Because of this phenomenon, tongue twisters are an interesting task that can provide insights into the compensatory strategies employed by speakers with partial glossectomy before and after the surgery. If the speakers with glossectomy allocate their resources to production speed, they should produce more errors than control speakers. On the other hand, if patients allocate their resources to accuracy, they should produce the tongue twisters more slowly than control speakers. It could be argued that the task instruction could influence a speaker's performance by emphasizing either speed or accuracy. In order to ensure consistent task performance, the 
participants in the present study were instructed to produce the tongue twisters quickly and not to worry about making errors. The expectation was that patients would produce more errors than control speakers.

Finally, it is possible that the presence of a lingual tumour and the subsequent glossectomy requires patients to allocate more resources to the phonological planning of the tongue twister because of the structural alteration of the tongue. This should result in longer speech planning times, i.e., longer pauses between tongue twister productions for speakers with glossectomy compared to control speakers. Note that due to the speed-accuracy trade-off, it would not be possible to distinguish the phonological planning from the motor execution of the task based on production speed and accuracy.

To assess the effect of the presence of a tumour as well as the glossectomy surgery on the patients' production of tongue twisters, the patients were examined before and after the surgery and compared to healthy control participants. The outcome measures were the patients' speech acceptability, rate of errors, the time needed to produce the tongue twisters, pauses between the item repetitions, and the tongue shape during the production of the consonants $[\mathrm{t}]$ and $[\mathrm{k}]$ before and after surgery. The outcome measures for control participants were the same as for patients, except that speech acceptability was not measured and that there was only a single recording session. The research aimed to test four hypotheses.

1. Compared to the controls, the patients' speech should be affected by the presence of the lingual tumour before surgery and by the partial resection of the tongue after surgery. As a result, patients should either demonstrate a higher rate of tongue twister-induced speech errors or show slower task execution than controls. . Since the patients were instructed to produce the tongue twisters quickly, it was expected that the error rate would be higher while the production speed would remain the same.

2. The tongue resection should lead to a deterioration in the patients' speech. After the partial glossectomy surgery, the patients should either demonstrate a higher rate of tongue twister-induced speech errors or a slower task execution than before the tongue resection. 
Since the patients were instructed to produce the tongue twisters quickly, it was expected that the error rate would be higher while the production speed would remain the same.

3. Compared to the controls, the patients should require more phonological planning resources to produce the tongue twisters due to the lingual tumour (before surgery) and due to partial resection of the tongue (after surgery). Patients' pause durations should therefore be longer than those for control participants, both before and after the partial glossectomy.

4. After the partial glossectomy surgery, the patients should require more phonological planning resources to produce the tongue twisters than before the tongue resection. As a result, pause duration between the item repetitions should increase after the surgery.

\section{METHODS}

\section{Participants}

Fifteen tongue cancer patients (10 male, 5 female) undergoing a small to medium lateral partial glossectomy took part in the study. Of these patients, 8 received local reconstructions and 7 received free flaps. The location and extent of the surgical resection was sketched by the operating surgeon using a graphical mapping protocol, based on work by Beck et al. (1998). The surgical drawings were digitized and the area sketched was measured and expressed as a percentage of the tongue surface in the horizontal plane (Bressmann et al., 2007, 2009). The participants were seen a few days before the operation and 6-9 weeks after the surgery, after wound healing was complete but before the onset of radiation therapy. An additional ten healthy control participants ( 1 male, 9 female) participated in the study.

Materials and recording procedure A popular technique used in tongue twister research is called "Spoonerisms of Laboratory Induced Predisposition” (SLIP, Motley \& Baars, 1976; Pouplier, 2007). The SLIP technique uses juxtaposed minimal pairs such as “cop top, kip tip," which are presented in different permutations in order to elicit speech errors. However, the SLIP technique requires a considerable number of repetitions and was not suited for the clinical patient group in the present study. In order to ensure that the task was manageable and could be completed in approximately 5 minutes, the study used naturalistic sentence level tongue twisters. The study focused on 
lingual targets, specifically the confusion of voiceless alveolar and velar plosive targets. All participants read three repetitions of two tongue twisters designed to elicit $[\mathrm{t}] /[\mathrm{k}]$ confusions: "Kate takes cakes to Tate," ("Kate" tongue twister) and "He takes Kate's cake to Tate" ("He" tongue twister). The participants were instructed to read the tongue twisters as quickly as possible and not to worry about making errors. The patients also read three phonetically varied 6word sentences from the computerized Test of Children's Speech (TOCS+; Hodge \& Gotzke, 2007; Gotzke \& Hodge, 2005) at a comfortable speed. The sentences were presented in a randomized order.

Midsagittal tongue movement was recorded with a General Electric Logiq Alpha 100 MP ultrasound scanner (General Electric Medical Systems, WI 53201) with a model E72 $6.5 \mathrm{MHz}$ transducer. Participants were seated on an office chair leaning their forehead against a head stabilizer and their neck on the ultrasound transducer (Rastadmehr et al., 2008). The ultrasound video output was recorded to a digital mini camcorder (ZR 45 MC, Canon Canada Inc., ON L5T 1P7) with a frame rate of $29.97 \mathrm{fps}$. The acoustic signal was recorded to the same video camera with an AKG C420 headset microphone (AKG Acoustics, TN 37217) and a Behringer UltraGain Pro 2200 line-driver (Behringer, WA 98011). The sampling rate was $44.1 \mathrm{kHz}$ with a signal resolution of 16 bit.

Speech acceptability

Speech acceptability measures the overall naturalness or the subjectively perceived competence of a speaker (Dagenais, Adlington \& Evans, 2011). For the analysis of the patients' speech acceptability, five naïve listeners listened to the phonetically varied TOCS+ sentences before and after the surgery using Telex 1210 headphones (Telex Communications, Inc., Burnsville, MN 55337). The order of presentation of the speakers and the sentences was randomized. The participants did not receive any specific training or instructions for the task. The listeners evaluated the speakers' speech acceptability on a 4 point scale of $0=$ normal, $1=$ mildly unacceptable, $2=$ moderately unacceptable, and $3=$ very unacceptable.

Perceptual and quantitative analysis of the tongue twisters 
The goal of the perceptual analysis of the tongue twisters was to identify the correct and incorrect productions of the $/ \mathrm{t} /$ and $/ \mathrm{k} /$ target sounds. In a first step, three research assistants transcribed the tongue twisters orthographically and annotated their transcriptions with observations if and where errors were perceived. In the second step, a senior research assistant with phonetic training summarized and reconciled the three transcriptions. In the third step, the third author compared the summaries to the listeners' evaluations and the original speech recordings and classified the speech errors into the categories $[\mathrm{t}] /[\mathrm{k}]$ confusion $([\mathrm{t}]$ confused with $[\mathrm{k}]$ or vice versa), sound substitution (e.g. $[\mathrm{t}]$ replaced with $[\mathrm{s}])$, elision $([\mathrm{t}]$ or $[\mathrm{k}]$ omitted), and other (e.g., sound distortion, voicing error). From the speech oscillogram (amplitude-time display), we measured the duration of each individual production of the tongue twisters. We also measured the duration of the pauses between the repetitions. The measurements were conducted by the third author. The pauses were clearly visible in the speech oscillogram. Measurements were considered accurate within $10 \mathrm{~ms}$, and no further assessment of measurement reliability was undertaken.

Analysis of ultrasound data

The ultrasound video films were downloaded to a computer and analyzed with the Ultrasonographic Contour Analyzer for Tongue Surfaces (Ultra-CATS, Bressmann et al., 2005; Rastadmehr et al., 2008). This software allows the experimenter to make semi-automatic measurements of the tongue height. Based on the distance markers in the ultrasound image, the software is calibrated so that pixel distances can be converted into millimetres. A computer mouse was used to trace the tongue shape during the target sounds $/ \mathrm{t} /$ and $/ \mathrm{k} /$ in the image frame before the release of the plosion. Image frames were chosen based on the speech oscillogram. The distance from the ultrasound transducer to the tongue surface was measured. The measurements were made along radiating gridlines at $5^{\circ}$ intervals on extracted still frames.

The position of the participants' head on the transducer sometimes varied in the anteriorposterior dimension. Since a concentric measurement grid was used, this variation could be accommodated by varying the $0^{\circ}$ centre line in the data set. The measurements were obtained in data columns that corresponded to the height values along the $5^{\circ}$ grid lines. In order to align the surfaces for comparison, the measurements for individual patients were arranged so that an equal 
number of columns were obtained to the right (front of the tongue) and the left (back of the tongue) of the $0^{\circ}$ centre gridline. In case an even number of columns was obtained, the supernumerary column was added to the front of the tongue. This data adjustment ensured proper comparisons of the different parts of the tongue across participants and across testing sessions.

\section{Statistical analysis}

Speech acceptability was evaluated with paired t-tests within participants and with an unpaired ttest between patients and control. Additional t-tests were calculated between the participants with glossectomies that had been reconstructed locally vs. with flaps. Speech error rates, speech duration and pause duration were analyzed using mixed effects models (Baayen \& Milin, 2010; Jaeger, 2008). The tongue shapes for the correct productions of $/ \mathrm{t} /$ and $/ \mathrm{k} /$ were compared using smoothing spline ANOVA (SSANOVA; Gu, 2013; Davidson, 2006).

\section{RESULTS}

\section{Speech acceptability}

Patients had an average speech acceptability of $0.86(\mathrm{sd}=0.6)$ before and $1.02(\mathrm{sd}=0.55)$ after the surgery, where a rating of 0 reflects normal and a rating of 1 reflects mildly unacceptable speech. A paired t-test showed that overall speech acceptability decreased significantly from before to after the surgery $(\mathrm{df}=14, \mathrm{t}=-2.35, \mathrm{p}<0.05)$. Ten of the fifteen individual patients had better speech acceptability ratings before compared to after the surgery. In addition, closure procedure marginally affected speech acceptability: Patients with local reconstructions had marginally better post-surgery speech acceptability (mean $=0.77, \mathrm{sd}=0.27$ ) than patients with free flaps (mean=1.32, sd=0.65; t-test: $\mathrm{df}=7.861, \mathrm{t}=-2.0862, \mathrm{p}=0.07)$, even though speech acceptability ratings were more similar for both groups before surgery (local: mean $=0.63, \mathrm{sd}=0.26$; flap: mean=1.12, $\mathrm{sd}=0.78$; t-test: $\mathrm{df}=7.145, \mathrm{t}=-1.5719, \mathrm{p}=0.15)$. Finally, percent resection showed a significant correlation with speech acceptability both before $\left(\mathrm{t}=2.552, \mathrm{p}<0.05\right.$, adjusted $\left.\mathrm{r}^{2}=0.28\right)$ and after $\left(t=2.844, p<0.05\right.$, adjusted $\left.r^{2}=0.34\right)$ the surgery. Since the effect of closure procedure was only marginal in the present group of patients, the data for the partial glossectomy patients were pooled in all further comparisons with the group of controls.

Speech error rates 
Tongue twisters pose the challenge to produce a difficult sequence of alternating sounds quickly and error-free. As discussed in the introduction, these two aspects represent a trade-off: The faster the tongue twister is produced the more errors the speaker is likely to make. Therefore, both error rates (this section) and production speed (next section) were explored. It was investigated whether patients produced more tongue twister-induced speech errors after compared to before the surgery and whether patients produced more errors than healthy controls. Table 1 presents the number and distribution of speech errors for patients (pre and post surgery) and controls. It shows that total errors ranged from $11 \%$ to $22 \%$ percent of productions, depending on the target sound, participant group, and tongue twister. The following analyses were limited to the overall number of errors and not grouped by error subtype. Statistical investigations of the effects of participant group (patient vs. control), closure procedure (local reconstruction vs. free flap), and session (pre vs. post) were undertaken because these were considered the most relevant factors. Information about the tongue twisters (Kate takes cakes to Tate vs. He takes Kate's cakes to Tate), the individual repetitions (1, 2, and 3), the target sounds ([t] vs. $[\mathrm{k}]$ ), and the error types (confusions, substitutions, elisions and other) may be found in Tables 1 and 2 .

[Table 1 about here]

Mixed logit models were fit to the data (Jaeger, 2008). These models are appropriate for binomial response variables, in this case error vs. no error. They also allow modeling subject and item random effects within the same analysis. To test whether patients produced more speech errors after compared to before surgery and whether closure procedure affected error rates, a model was fit with t/k-production type (error vs. no error) as response variable, session (pre vs. post surgery), closure procedure (local reconstruction vs. free flap) and the interaction term as fixed effects, and subjects and items as random effects (model 1). Redundant fixed factors were removed from the model. Random slopes were added if they improved model fit (cf. Barr et al., 2013). The final model revealed a reliable effect of session (estimate $=0.14743, \mathrm{z}=2.024, \mathrm{p}<0.05$ ), such that patients produced significantly more errors after $(18.8 \%)$ than before $(15.7 \%)$ the surgery, and a reliable effect of closure procedure (estimate $=0.37238, \mathrm{z}=1.991, \mathrm{p}<0.05$ ), such that patients who received local reconstructions made fewer errors $(13.4 \%)$ than patients who 
received free flaps $(21.7 \%)$. To test whether patients produced more speech errors than control participants, separate models were fit for each session (pre vs. post surgery) with $\mathrm{t} / \mathrm{k}$-production type (error vs. no error) as response variable, group (patient vs. control) as fixed effect, and subjects and items as random effects (models 2 and 3). Neither model revealed a significant effect of group (estimate $=0.1121, \mathrm{z}=0.648, \mathrm{p}=0.517$ before surgery and estimate $=-0.04476, \mathrm{z}=-$ $0.276, \mathrm{p}=0.782$ post surgery). Patients' error rates ( $15.7 \%$ pre and $18.8 \%$ post surgery) did not differ from those of control participants $(17.4 \%)$.

\section{Duration measurements}

It was tested whether patients took longer to produce the tongue twisters after compared to before the surgery and whether patients had longer production times than healthy controls. Production durations ranged from $969 \mathrm{~ms}$ to $3294 \mathrm{~ms}$. Two further productions were considered to be outliers (> $4000 \mathrm{~ms}$ ) and were removed from the dataset. Table 2 presents the average production durations of each tongue twister repetition for patients (pre and post surgery) and controls. The table shows that patients consistently slowed down throughout the task, such that they produced the first rendition of each twister faster than the second and the second rendition faster than the third. Control participants, on the other hand, produced the second rendition of each twister faster than the first and the first rendition faster than the third. The table also suggests that control participants produced the tongue twisters faster than patients.

[Table 2 about here]

Mixed models were fit to the data (Baayen \& Milin, 2010) so that subject and item random effects could be modeled within the same analysis. To test whether patients produced the tongue twisters more slowly after compared to before surgery and whether production speed was affected by closure procedure, a model was fit with production duration in milliseconds as the response variable. Fixed and random effects and the model comparison procedure were the same as for model 1 above. The final model did not include any fixed factors. The analysis showed that patients did not produce the tongue twisters more slowly after (average $1796 \mathrm{~ms}$ ) compared to before surgery (average $1832 \mathrm{~ms}$ ). In fact, patients were numerically faster in producing the tongue twisters after compared to before the surgery. To test whether patients produced the 
tongue twisters more slowly than control participants, separate models for each session were fit (pre vs. post surgery) with production duration in milliseconds as response variable, and the same fixed and random effects as in models 2 and 3 above. Both models revealed a significant effect of group (estimate $=125.83, \mathrm{t}=2.947, \mathrm{p}<0.01$ pre surgery and estimate $=106.00, \mathrm{t}=2.37$, $\mathrm{p}<0.01$ post surgery), indicating that the patients were slower than the control participants (average $1580 \mathrm{~ms}$ ) in their production of tongue twisters, both before (average $1832 \mathrm{~ms}$ ) and after surgery (average $1796 \mathrm{~ms}$ ).

In a next step, it was investigated whether patients produced longer pauses between tongue twisters after compared to before the surgery and whether patients produced longer pauses than healthy controls. Pause durations ranged from $10 \mathrm{~ms}$ to $952 \mathrm{~ms}$. Four further productions were considered to be outliers (> $1500 \mathrm{~ms}$ ) and were removed from the dataset. Table 2 presents the average pause durations between renditions one and two as well as two and three of each tongue twister for patients (pre and post surgery) and controls. The table suggests that patients produced longer pauses between tongue twisters than control participants.

To test whether patients produced longer pauses between tongue twisters after compared to before surgery, a model was fit with pause duration in milliseconds as response variable. Again, fixed and random effects and the model comparison procedure were the same as for model 1 above The final model revealed a marginal effect of session (estimate $=30.73, \mathrm{t}=1.936$, $\mathrm{p}=0.0558$ ), indicating that there was a trend for patients to produce shorter pauses after ( mean=237 ms, sd=172) compared to before (mean=302 ms, sd=210) the surgery. In addition, there was a marginal effect of closure procedure (estimate $=53.92, \mathrm{t}=2.122, \mathrm{p}=0.0533$ ), such that patients who received a free flap produced marginally shorter (mean=212 ms, $s d=176$ ) pauses than patients who received a local closure (mean=322 ms, sd=196). To test whether patients produced longer pauses between tongue twisters than control participants, separate models were fit for each session (pre vs. post surgery) with pause duration in milliseconds as response variable, group (patient vs. control) as fixed effect, and subjects and items as random effects. Both models revealed a significant effect of group (estimate $=95.813, \mathrm{t}=3.668, \mathrm{p}<0.01$ pre surgery and estimate $=67.298, \mathrm{t}=2.813, \mathrm{p}<0.05$ post surgery), indicating that patients paused 
longer than control participants (average $109 \mathrm{~ms}$ ) between tongue twisters, both before (average $302 \mathrm{~ms}$ ) and after surgery (average $237 \mathrm{~ms}$ ).

Averaged tongue shapes

An analysis of patients' tongue shapes during the productions of the two tongue twisters was undertaken. Figures 1 and 2 show the averaged tongue shapes for productions of [k] and [t] before and after the operation. The figures show that the tongue tended to be higher in the oral cavity after compared to before the surgery.

SSANOVA (Gu 2013) was used to analyze the tongue shapes for [k] and [t] before and after the surgery for patients who received local reconstructions and patients who received free flaps. SSANOVA analyses compare two sets of curves and determine whether they are statistically significantly different. Such analyses have been used by several ultrasound researchers to analyze images of tracings of the tongue (e.g. Davidson, 2006, Mielke, Baker, Archangeli, 2010, Mielke, Olson, Baker, Archangeli, 2011). To compare two sets of curves, an SSANOVA generates smooth lines and $95 \%$ confidence intervals for each set. Two sets of curves are considered to differ significantly in all areas where the confidence intervals of the two smooth lines do not overlap. Thus, an SSANOVA analysis captures which points on the length of the curve differ reliably.

The SSANOVA analyses depicted in Figures 1 and 2 show that the body of the tongue was reliably higher after compared to before surgery for patients who received a free flap for both the production of $[\mathrm{k}]$ and $[\mathrm{t}]$. In contrast, there was no such consistent pattern for patients who received local reconstructions.

\section{DISCUSSION}

A partial glossectomy is a significant and often debilitating surgery that can affect speech, as demonstrated by the significant decrease in speech acceptability in the present study. This effect was noted before and after the surgery for the patients: The moderate but significant correlations between percent resection and speech acceptability before surgery suggests that the presence and size of the tumour affected speech acceptability. After the surgery, the moderate but significant correlations between percent resection and speech acceptability showed that the extent of the 
resection impacted speech acceptability. This mirrors previous findings (Nicoletti et al., 2004; Bressmann et al., 2009). It should be noted that the extent of the resection is determined by the size of the tumour. It should also be noted that resections that are reconstructed with a flap tend to be larger than local closures. This may account for the marginally poorer speech acceptability for the patients with the flap reconstructions.

Four hypotheses were tested in this study. The first two hypotheses concerned the speedaccuracy trade-off and the patients' compensatory strategies. Since all participants were instructed to focus on speed of production, the patients were expected to show a higher error rate but the same duration of production. In particular, it was expected that patients would produce more errors than controls ( $1^{\text {st }}$ hypothesis) and more errors after surgery compared to before surgery ( $2^{\text {nd }}$ hypothesis), whereas production duration differences between patients and controls $\left(1^{\text {st }}\right.$ hypothesis) and after compared to before surgery $\left(2^{\text {nd }}\right.$ hypothesis $)$ were not expected.

The logit analysis of the rate of speech errors indicated that the patients did not differ significantly from the controls with regards to the speech error rate. However, the patients' error rate increased significantly after the glossectomy surgery compared to before, and patients with flap reconstructions made more errors than patients with local closures. The result that patients did not differ from controls even though their error rate increased significantly after compared to before the surgery was observed because the patients' error rates were numerically lower than the controls' error rates before surgery and numerically higher than the controls' error rates after surgery. The analysis of production duration revealed that the patients produced the tongue twisters significantly more slowly than the controls. However, there were no significant differences between the production speed of the tongue twisters by the glossectomy patients before and after the surgery. Therefore, the $1^{\text {st }}$ hypothesis (patients would produce more errors at similar production durations than controls) could not be confirmed, whereas the $2^{\text {nd }}$ hypothesis (patients would produce more errors at similar production durations after surgery compared to before) was confirmed.

These results can be interpreted in terms of a speed-accuracy trade-off: Contrary to expectation, the patients did not differ from the controls with regards to their error rates. Instead, the patients 
produced the tongue twisters more slowly than the controls. This could be a consequence of articulatory gestures that were hampered by the tumour and the subsequent resection and reconstruction. However, Rastadmehr et al. (2008) found that patients were able to increase the speed of their tongue movement after a partial glossectomy. A possible alternative explanation is that patients may have focused more on accuracy despite the instructions to focus on production speed. The patients may have produced the tongue twisters significantly more slowly than controls in order to maintain error rates similar to those of controls. Finally, it is also possible that patients may have taken more time for phonological planning. The difference in duration across repetitions for patients and controls may lend weight to this interpretation. The control speakers produced progressively faster repetitions while the glossectomy patients slowed down over the repetitions, possibly in order to maintain accuracy of the productions. Comparing the results within participants, the patients produced more errors after compared to before the surgery, while the glossectomy surgery did not affect how quickly patients produced the tongue twisters. Thus, maintaining the speed of production after surgery probably resulted in more errors because the resection and reconstruction had affected the patients' articulatory gestures.

The 3rd and 4th hypotheses considered phonological planning and suggested that patients would produce longer pauses between tongue twisters than control participants ( $3^{\text {rd }}$ hypothesis) and longer pauses between tongue twisters after surgery compared to before ( $4^{\text {th }}$ hypothesis). The $3^{\text {rd }}$ hypothesis was confirmed: Patients produced consistently longer pauses between tongue twisters than control participants. The presence of a lingual tumour and the subsequent glossectomy may require patients to pay more attention to the phonological planning of the utterance, which may be reflected in the increased pause length. The $4^{\text {th }}$ hypothesis was not confirmed: Based on the brain imaging research by Mosier et al. (2005), it was expected that the increase in activity in the brain's speech motor centres after the glossectomy surgery would be reflected in an increase in the pause durations. However, the patients did not produce longer pauses between the tongue twisters after the surgery compared to before.

The quantitative analysis of tongue shapes is a challenging task because tongue shapes are individually varied. Davidson et al. (2006) suggested a smoothing spline ANOVA method (Gu, 2013) to analyze deviations of tongue shapes between sound realizations. In the present study, 
this method was applied to the glossectomy patients' data to demonstrate the quantitative differences between the realizations of the $[\mathrm{t}]$ and $[\mathrm{k}]$ sounds before and after the partial tongue resection. The observed difference in average height of the tongue after the operation has previously been documented by Rastadmehr et al. (2008) who speculated that increased speed and height of tongue movement could be an indicator of articulatory compensation on the patients' part. In the present study, this effect was observed for the patients who had a flap reconstruction. The patients with local reconstructions had more mixed results with regards to postoperative tongue height. However, it should be noted that, despite the statistical significance, the differences in tongue height were small. While the structural change of the partially resected tongue with the ensuing flap reconstruction may be a plausible reason for this effect, it cannot be ruled out that a higher tongue position after the surgery could be due to natural phonetic variation. Since there were not two separate data collection sessions for the controls, this possibility cannot be dismissed. It should also be noted that the tongue twisters were produced at a fast speaking rate which tends to reduce movement excursion (Ostry \& Munhall, 1985).

Finally, there were a number of possible shortcomings of the present study. The ultrasound data were acquired with the normal NTSC frame rate of $29.97 \mathrm{fps}$, which was considered fast enough for the plosives studied. However, this frame rate does not provide the movement detail that a faster ultrasound system could provide. The alignment of the data around the $0^{\circ}$ gridline was an effective way of ensuring that the corresponding anatomical segments of the tongues of different speakers were compared. However, individual oral cavities and tongues vary in their size and shape, so it is impossible to determine without an external frame of reference how accurate such a match is.

The patients were seen 6-9 weeks after the operation, which is relatively early after the operation. At this time wound healing was complete but adjuvant radiation therapy had not started, yet. Since the radiation therapy can induce additional tissue irritation, swelling and necrosis, there was no alternative to the time line of the post-operative data recording. A case study by Kaipa et al. (2012) demonstrated that a patient's vowel space and consonant clarity may undergo changes in the months following the surgery. In the present study, it was not possible to determine whether all patients had reached a stable speech outcome, yet. 
The tongue twisters used in the present research were constructed to elicit $[\mathrm{t}] /[\mathrm{k}]$ errors. The level of difficulty of these tongue twisters was considered quite challenging. This approach did not allow making the detailed observations that can be obtained with a method such as the SLIP technique (Pouplier, 2007). Nevertheless, the tongue twisters were successful in that a number of speech errors could be elicited in a convenient and naturalistic manner in both the patient and the control speakers.

\section{CONCLUSION}

A tongue twister is a demanding task for the speech production system, possibly even more so when this system has been affected by a surgical resection such as a glossectomy surgery. The results of the present study demonstrated that the surgical resection of the tongue changed the patients' error rate but not the speed of production. However, compared to control participants, patients were consistently slower in producing the tongue twisters and made longer pauses between tongue twister repetitions. The results suggest that patients' altered tongue anatomy not only affects the production of a tongue twister but also the phonological planning of the sound sequence. 


\section{REFERENCES}

Baayen, R. H. \& Milin, P. (2010). Analyzing reaction times. International Journal of Psychological Research, 3.2, 12-28 .

Barr, D. J., Levy, R., Scheepers, C., \& Tily, H. J. (2013). Random effects structure for confirmatory hypothesis testing: Keep it maximal. Journal of Memory and Language, 68, 255278

Beck, J.M., Wrench, A., lackson, M., Soutar, D., Robertson, A.G., \& Laver, J. (1998). Surgical mapping and phonetic analysis in intra-oral cancer. In W. Ziegler \& K. Deger (Eds): Clinical Phonetics and Linguistics, London: Whurr, pp. 481-492.

Bressmann, T., Jacobs, H., Quintero, J., \& Irish, J.C. (2009). Speech outcomes for partial glossectomy surgery: Measures of speech articulation and listener perception. Canadian Journal of Speech-Language Pathology and Audiology, 33 (4), 204-210.

Bressmann, T., Ackloo, E., Heng, C.-L., \& Irish, J.C. (2007). Quantitative three-dimensional ultrasound imaging of partially resected tongues. Otolaryngology - Head and Neck Surgery, 136 (5), 799-805.

Bressmann, T., Heng, C.-L., \& Irish, J.C. (2005). Applications of 2D and 3D ultrasound imaging in speech-language pathology. Journal of Speech-Language Pathology and Audiology, 29 (4), 158-168.

Dagenais, P.A., Adlington, L.M., \& Evans, K.J. (2011). Intelligibility, comprehensibility, and acceptability of dysarthric speech by older and younger listeners. Journal of Medical SpeechLanguage Pathology, 19 (4), 37-48.

Davidson, L. (2006). Comparing tongue shapes from ultrasound imaging using smoothing spline analysis of variance. Journal of the Acoustical Society of America, 120 (1), 407-415. 
Goldstein, L., Pouplier, M., Chen, L., Saltzman, E., \& Byrd, D. (2007). Dynamic action units slip in speech production errors. Cognition, 103 (3), 386-412.

Gotzke, C.L. \& Hodge, M.M. (2005). Relationship between measures of intelligibility and phonetic accuracy in children with and without cleft palate. Canadian Acoustics - Acoustique Canadienne, 33 (3), 80-81.

Gu C. (2013). Smoothing Spline ANOVA Models (2nd Ed.). New York: Springer-Verlag.

Hodge, M. \& Gotzke, C.L. (2007). Preliminary results of an intelligibility measure for englishspeaking Children with Cleft Palate. Cleft Palate-Craniofacial Journal, 44 (2), 163-174.

Jaeger, T.F. (2008). Categorical data analysis: Away from ANOVAs (transformation or not) and towards logit mixed models. Journal of Memory and Language, 59 (4), 434-446.

Kimata, Y., Sakuraba, M., Hishinuma, S., Ebihara, S., Hayashi, R., Asakage, T., Nakatsuka, T., \& Harii, K. (2003). Analysis of the relations between the shape of the reconstructed tongue and postoperative functions after subtotal or total glossectomy. Laryngoscope, 113 (5), 905-909.

Konstantinovic, V.S., \& Dimic, N.D. (1998). Articulatory function and tongue mobility after surgery followed by radiotherapy for tongue and floor of the mouth cancer patients. British Journal of Plastic Surgery, 51 (8), 589-593.

McMillan, C.T., \& Corley, M. (2010). Cascading influences on the production of speech: Evidence from articulation. Cognition, 117 (3), 243-260.

Mielke, J., Olson, K.S., Baker, A., \& Archangeli, D. (2011). Articulation of the Kagayanen interdental approximant: An ultrasound study. Journal of Phonetics, 39 (3), 403-412. 
Mielke, J., Baker, A., \& Archangeli, D. (2010). Variability and homogeneity in American English /r/ allophony and /s/ retraction. In Lab Phon 10: Variation, Detail, and Representation. C. Fougeron \& N. Nguyen (Eds). Berlin: Mouton de Gruyter, pp. 699-719.

Motley, M. T., \& Baars, B. J. (1976). Laboratory induction of verbal slips: A new method for psycholinguistic research. Communication Quarterly, 24 (2), 28-34.

Murano, E.Z., Shinagawa, H., Zhuo, J., Gullapalli, R.P., Ord, R.A., Prince, J.L., \& Stone, M. (2010). Application of diffusion tensor imaging after glossectomy. Otolaryngology - Head and Neck Surgery, 143 (2), 304-306.

Nicoletti, G., Soutar, D.S., Jackson, M.S., Wrench, A.A., Robertson, G., \& Robertson, C. (2004). Objective assessment of speech after surgical treatment for oral cancer: Experience from 196 selected cases. Plastic and Reconstructive Surgery, 113 (1), 114-125.

Oppenheim, G.M. \& Dell, G.S. (2010). Motor movement matters: The flexible abstractness of inner speech. Memory and Cognition, 38 (8), 1147-1160.

Oppenheim, G.M. \& Dell, G.S. (2008). Inner speech slips exhibit lexical bias, but not the phonemic similarity effect. Cognition, 106 (1), 528-537.

Ostry, D.J. \& Munhall, K.G. (1985). Control of rate and duration of speech movements. Journal of the Acoustical Society of America, 77 (2), 640-648.

Pouplier, M. (2007). Tongue kinematics during utterances elicited with the SLIP technique. Language and Speech, 50 (3), 311-341.

Pouplier, M. \& Hardcastle, W. (2005). A re-evaluation of the nature of speech errors in normal and disordered speakers. Phonetica, 62 (2-4), 227-243. 
Rastadmehr, O., Bressmann, T., Smyth, B., \& Irish, J.C. (2008). Increased midsagittal tongue velocity as indication of articulatory compensation in patients with lateral partial glossectomies. Head and Neck, 30 (6), 718-726.

Whitehill, T.L., Ciocca, V., Chan, J.C.-T., \& Samman, N. (2006). Acoustic analysis of vowels following glossectomy. Clinical Linguistics and Phonetics, 20 (2-3), 135-140. 
Table 1: Number and distribution of speech errors. $\mathrm{N}$ provides the total number of $\mathrm{t} / \mathrm{k}$ productions.

\begin{tabular}{|c|c|c|c|c|c|c|c|c|}
\hline Group & Tongue twister & $\begin{array}{l}\text { Target } \\
\text { sound }\end{array}$ & $\mathrm{N}$ & Total errors & Confusions & Substitutions & Elisions & Other \\
\hline \multirow[t]{4}{*}{ Pre-operative } & Kate takes cakes & {$[\mathrm{t}]$} & 233 & $54(23 \%)$ & $14(6 \%)$ & $6(3 \%)$ & $27(12 \%)$ & $7(3 \%)$ \\
\hline & to Tate. & {$[\mathrm{k}]$} & 188 & $22(12 \%)$ & $8(4 \%)$ & $5(3 \%)$ & $1(1 \%)$ & $8(4 \%)$ \\
\hline & He takes Kate's & {$[\mathrm{t}]$} & 234 & $28(12 \%)$ & $8(3 \%)$ & $6(3 \%)$ & $6(3 \%)$ & $8(3 \%)$ \\
\hline & cakes to Tate. & {$[\mathrm{k}]$} & 187 & $28(15 \%)$ & $2(1 \%)$ & $4(2 \%)$ & $17(9 \%)$ & $5(3 \%)$ \\
\hline Pre-operative total & & & 842 & $132(16 \%)$ & $32(4 \%)$ & $21(2 \%)$ & $51(6 \%)$ & $28(3 \%)$ \\
\hline \multirow[t]{4}{*}{ Post-operative } & Kate takes cakes & {$[\mathrm{t}]$} & 233 & $45(19 \%)$ & $7(3 \%)$ & $1(0 \%)$ & $32(14 \%)$ & $5(2 \%)$ \\
\hline & to Tate. & {$[\mathrm{k}]$} & 186 & $39(21 \%)$ & $10(5 \%)$ & $15(8 \%)$ & $8(4 \%)$ & $6(3 \%)$ \\
\hline & He takes Kate's & {$[\mathrm{t}]$} & 228 & $25(11 \%)$ & $15(7 \%)$ & $0(0 \%)$ & $9(4 \%)$ & $1(0 \%)$ \\
\hline & cakes to Tate. & {$[\mathrm{k}]$} & 184 & $47(26 \%)$ & $9(5 \%)$ & $11(6 \%)$ & $23(13 \%)$ & $4(2 \%)$ \\
\hline Post-operative total & & & 831 & $156(19 \%)$ & $41(5 \%)$ & $27(3 \%)$ & $72(9 \%)$ & $16(2 \%)$ \\
\hline \multirow[t]{3}{*}{ Controls } & Kate takes cakes & {$[\mathrm{t}]$} & 154 & $33(21 \%)$ & $9(6 \%)$ & $2(1 \%)$ & $19(12 \%)$ & $3(2 \%)$ \\
\hline & to Tate. & {$[\mathrm{k}]$} & 125 & $14(11 \%)$ & $4(3 \%)$ & $0(0 \%)$ & $5(4 \%)$ & $5(4 \%)$ \\
\hline & He takes Kate's & {$[\mathrm{t}]$} & 155 & $29(19 \%)$ & $15(10 \%)$ & $1(1 \%)$ & $10(6 \%)$ & $3(2 \%)$ \\
\hline
\end{tabular}


cakes to Tate.

Controls total [k]

135

569
$23(17 \%)$

$99(18 \%)$

$$
9(7 \%)
$$

$37(7 \%)$
$0(0 \%)$

$3(1 \%)$
$4(3 \%)$

$38(7 \%)$
$10(7 \%)$

$21(4 \%)$ 
Table 2: Mean production durations of the tongue twisters and mean pause durations between tongue twisters for each participant group, tongue twister, and repetition.

\begin{tabular}{|c|c|c|c|c|}
\hline Group & Tongue twister & Repetition & Mean production duration (ms) & Mean pause duration (ms) \\
\hline \multirow[t]{6}{*}{ Pre-operative } & Kate takes cakes & 1 & $1575(\mathrm{sd}=156)$ & - \\
\hline & \multirow[t]{2}{*}{ to Tate. } & 2 & $1743(\mathrm{sd}=336)$ & $372(s d=276)$ \\
\hline & & 3 & $1923(\mathrm{sd}=345)$ & $270(s d=204)$ \\
\hline & He takes Kate's & 1 & $1704(\mathrm{sd}=170)$ & - \\
\hline & \multirow[t]{2}{*}{ cakes to Tate. } & 2 & $1994(\mathrm{sd}=437)$ & $288(\mathrm{sd}=204)$ \\
\hline & & 3 & $2058(\mathrm{sd}=287)$ & $283(\mathrm{sd}=147)$ \\
\hline \multicolumn{3}{|c|}{ Pre-operative mean } & $1832(\mathrm{sd}=341)$ & $302(\mathrm{sd}=210)$ \\
\hline \multirow[t]{6}{*}{ Post-operative } & Kate takes cakes & 1 & $1615(\mathrm{sd}=316)$ & - \\
\hline & \multirow[t]{2}{*}{ to Tate. } & 2 & $1707(\mathrm{sd}=381)$ & $308(s d=200)$ \\
\hline & & 3 & $1755(\mathrm{sd}=250)$ & $207(\mathrm{sd}=129)$ \\
\hline & He takes Kate's & 1 & $1741(\mathrm{sd}=165)$ & - \\
\hline & \multirow[t]{2}{*}{ cakes to Tate. } & 2 & $1948(\mathrm{sd}=389)$ & $185(\mathrm{sd}=166)$ \\
\hline & & 3 & $2008(\mathrm{sd}=306)$ & $255(\mathrm{sd}=178)$ \\
\hline \multicolumn{3}{|c|}{ Post-operative mean } & $1796(\mathrm{sd}=332)$ & $237(\mathrm{sd}=172)$ \\
\hline \multirow[t]{2}{*}{ Controls } & Kate takes cakes & 1 & $1442(\mathrm{sd}=368)$ & - \\
\hline & to Tate. & 2 & $1384(\mathrm{sd}=301)$ & $91(\mathrm{sd}=29)$ \\
\hline
\end{tabular}




$\begin{array}{llll} & 3 & 1501(\mathrm{sd}=306) & 143(\mathrm{sd}=45) \\ \text { He takes Kate's } & 1 & 1706(\mathrm{sd}=496) & - \\ \text { cakes to Tate. } & 2 & 1579(\mathrm{sd}=271) & 47(\mathrm{sd}=15) \\ & 3 & 1868(\mathrm{sd}=615) & 110(\mathrm{sd}=35) \\ & & 1580(\mathrm{sd}=428) & 109(\mathrm{sd}=110)\end{array}$




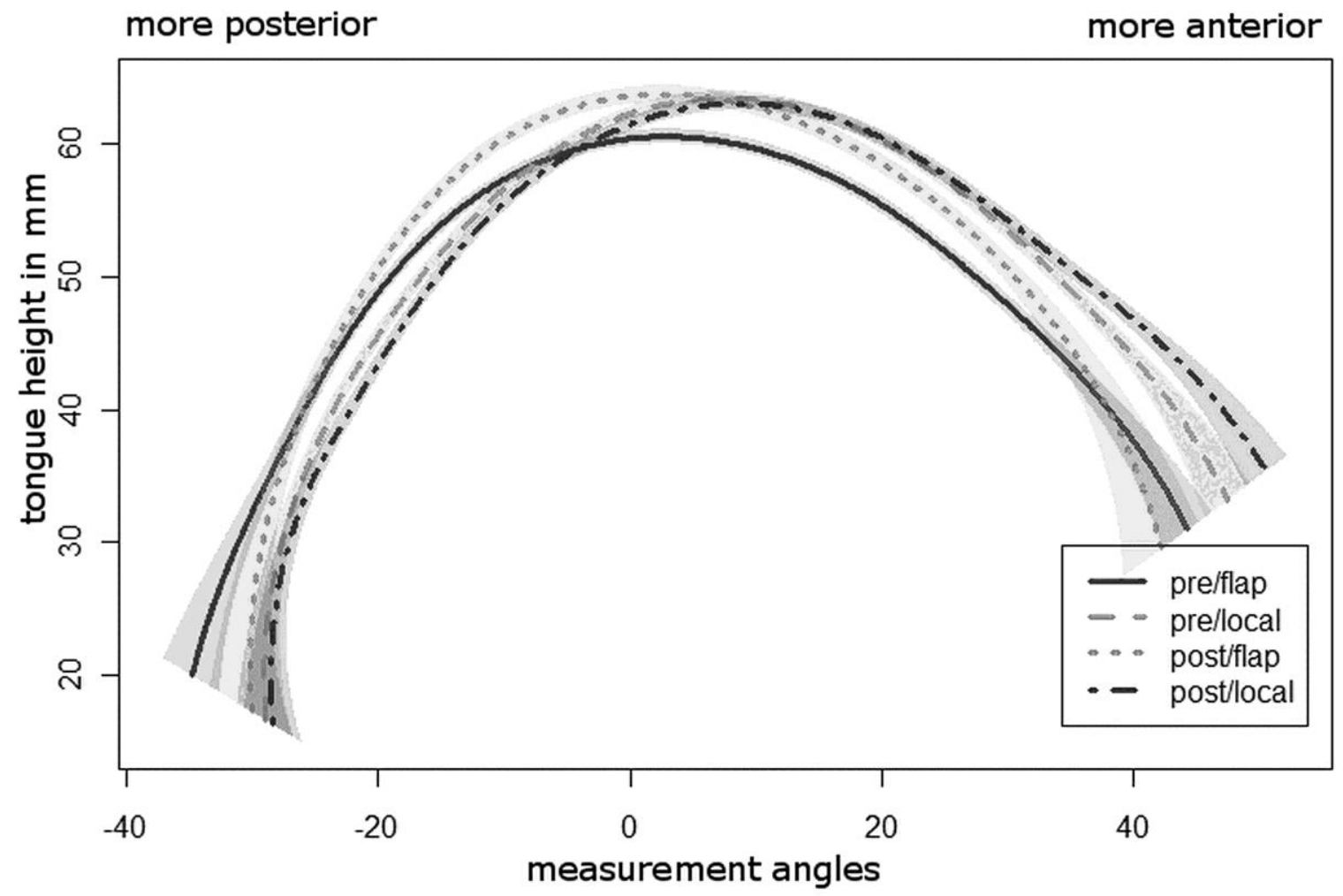

Figure 1. Averaged tongue shapes during the production of $[\mathrm{k}]$ before and after the surgery for the patients who received local reconstructions and for the patients who received free flaps. The $x$-axis displays the height measurements along the radiating grid lines in the Ultra-CATS software. 


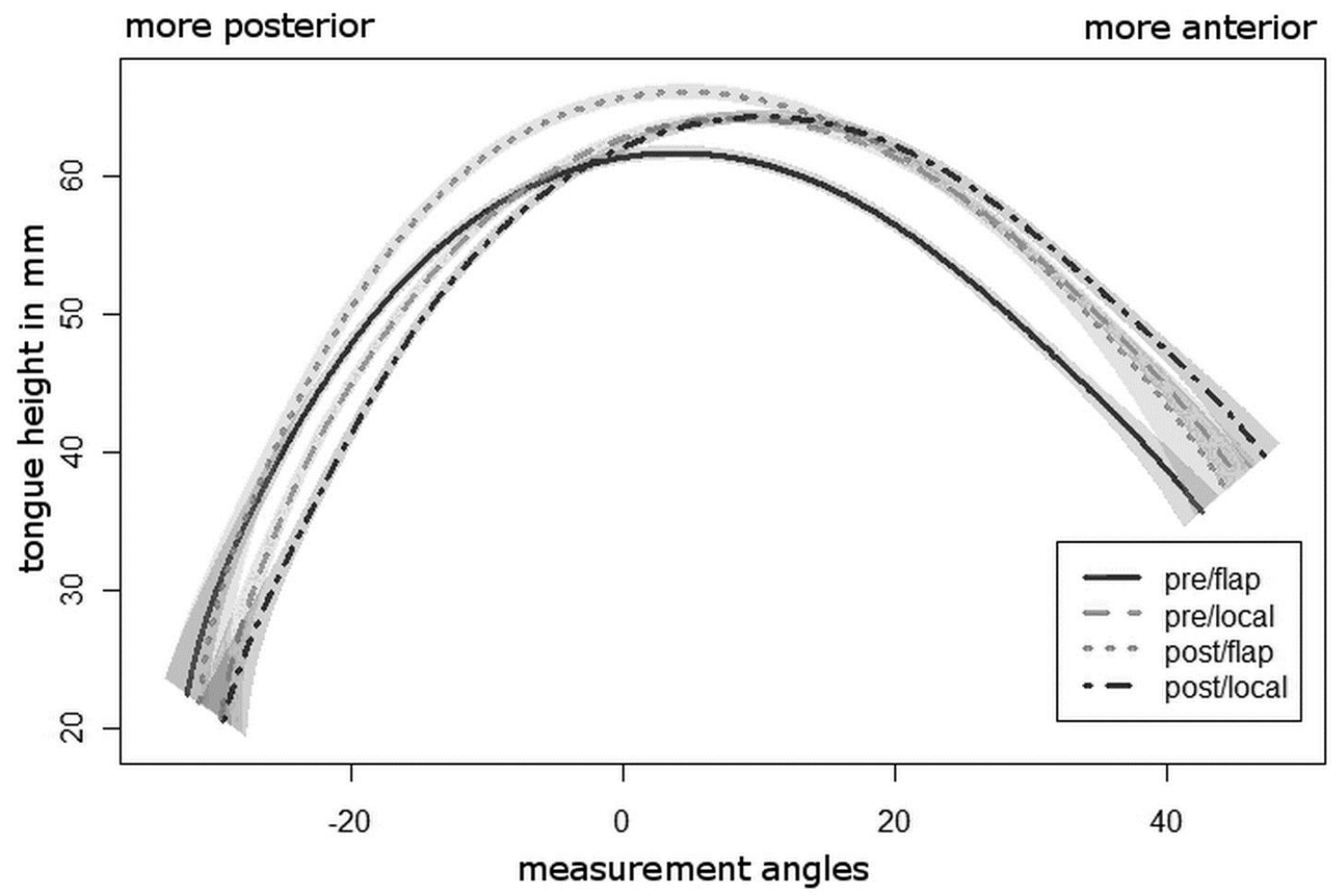

Figure 2. Averaged tongue shapes during the production of $[\mathrm{t}]$ before and after the surgery for the patients who received local reconstructions and for the patients who received free flaps. The $x$ axis displays the height measurements along the radiating grid lines in the Ultra-CATS software. 